\title{
Primary Amoebic Meningoencephalitis: First Reported Case from Rohtak, North India
}

\author{
Naveen Gupta ${ }^{1}$, Hemlata Bhaskar ${ }^{2}$, Shalini Duggal ${ }^{3}$, Pratap S. Ghalaut ${ }^{2}$, Shailja Kundra ${ }^{4}$ and Des R. Arora ${ }^{2}$ \\ ${ }^{1}$ National Institute of Communicable Diseases, New Delhi; ${ }^{2}$ Post Graduate Institute of Medical Sciences, Rohtak; ${ }^{3}$ Ram Manohar Lohia \\ Hospital, New Delhi; ${ }^{4}$ Sunder Lal Jain Hospital, Delhi;
}

\begin{abstract}
A fatal case of primary amoebic encephalitis (PAM) in a 20 year old boy, a proven case of acute leukemic leukemia (ALL) type $L 2$, in remission is described. No history of swimming could be elicited. The clinical presentation, the isolation of the amoeba from the cerebrospinal fluid (CSF), the poor response to amphotericin B, and the ultimately fatal outcome are all consistent with the diagnosis of PAM. On the basis of its ability to grow at temperature $42^{\circ} \mathrm{C}$ and $45^{\circ} \mathrm{C}$, morphology of trophozoite, and the presence of flagellate forms in CSF, the amoeba was identified as Naegleria fowleri. Other drugs used in combination with amphotericin B are tetracycline, rifampicin, and miconazole. A possibility of PAM should always be considered in all cases of acute purulent meningoencephalitis in which no bacteria or fungus are found.

Key-Words: Naegleria, meningoencephalitis, fatal.
\end{abstract}

Primary amoebic meningoencephalitis (PAM) is a fulminant, often rapidly fatal infection affecting individuals with a recent history of swimming in warm water. It is caused by a free living amoeba Naegleria fowleri, an organism ubiquitous in soil and fresh water habitats [1]. It was first described by Fowler and Carter from Southern Australia in 1965, who found these in four autopsied patients [2]. The disease appears to be uniformly fatal and at present there is no standard regimen of therapy. Amphotericin B, tetracycline, rifampicin, and miconazole have been reported to have in vitro growth- inhibitory activity against this organism [3,4]. Approximately 310 cases of PAM have been reported internationally, mostly from United States, Australia and Europe. There are only seven survivors of PAM reported in Western Literature [5-11]. From India, only two survivors of Naegleria meningitis have been reported so far [12,13].

\section{Case Report}

A 20 year old male was admitted to a tertiary care institute in North India with history of fever, headache, loss of vision, hearing loss, slurring of speech, difficulty in swallowing and retention of urine for two days. All the relevant history of tuberculosis, diabetes mellitus and hypertension was insignificant. Prior to this illness he was a proven case of acute leukemic leukemia (ALL) type L2. He was put on standard chemotherapy consisting of adriamycin, vincristine, methotrexate and prednisolone and palliative; external radiotherapy to cranium, chest and neck on account of supraclavicular lymphadenopathy and mediastinal mass. The patient was under close follow-up and went in remission phase (proved by bone marrow aspiration) and was put on maintenance therapy.

Examination on admission revealed a young boy with a markedly stiff neck and a temperature of $38.5^{\circ} \mathrm{C}$. He was oriented, responded to painful stimuli and the limbs were hypertonic but no focal neurological signs were present.

Received on 20 February 2009; revised 13 June 2009.

Address for correspondence: Dr. Naveen Gupta. Assistant Director, Virology Division. National Institute of Communicable Diseases, New Delhi. E-mail: nicdnaveen@gmail.com. Phone: 9818771770.

The Brazilian Journal of Infectious Diseases 2009;13(3):236-237. (C) 2009 by The Brazilian Journal of Infectious Diseases and Contexto Publishing. All rights reserved.
Ophthalmological examination revealed keratitis, mild glaucomatous cupping, early papilloedema and left lateral rectus palsy. The CT scan head showed no abnormality.

Laboratory studies showed hemoglobin of $9 \mathrm{~g} / \mathrm{dL}$, hematocrit $39 \%$ and a leucocyte count of $12,000 / \mathrm{mm}^{3}$ with $80 \%$ neutrophils, $17 \%$ lymphocytes and $3 \%$ monocytes. Blood culture was sterile. The cerebrospinal fluid (CSF) was slightly turbid with a leukocyte count of $1,500 / \mathrm{mm}^{3}$ with a predominance of neutrophils (>90\%) and few lymphocytes. CSF protein was $56 \mathrm{mg} / \mathrm{dL}$ and sugar $185 \mathrm{mg} / \mathrm{dL}$. While the microbiological processing was done, patient was empirically treated with injection ceftriaxone, amikacin, mannitol considering the possibility of pyogenic meningitis. Gram stain, stain for acid fast bacilli, India ink preparations and cultures for bacteria and fungi were all negative. However, wet mount examination of the centrifuged CSF sediment revealed pus cells, a few red blood cells and numerous trophozoites with size approximately that of neutrophils. Presumptive identification of $N$. fowleri was made and intravenous amphotericin B (1.5mg/kg body weight) was started. The CSF was subsequently cultured on non nutrient agar with a lawn culture of Escherichia coli, as food for the parasite. A luxurious growth of amoebae was seen after 48 hours of incubation at $37^{\circ} \mathrm{C}$ (Figure $1 \mathrm{~A}$ and $\mathrm{B}$ ). Identification of $N$. fowleri was determined with following characteristics:

a) Ability to grow at $42^{\circ} \mathrm{C}$ and $45^{\circ} \mathrm{C}$

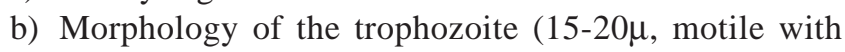
eruptive pseudopodia and a large nucleus).

c) Morphology of flagellate form (exposing trophozoite to sterile distilled water and incubation at $42^{\circ} \mathrm{C}$ ).

d) Morphology of cyst (10-12 $\mu$, spherical and smooth walled)

The patient showed some improvement after 3 days and able to take liquid diet and obey a few commands.

Subsequently he started deteriorating, level of consciousness decreased and neck stiffness increased and temperature rose to $39.0^{\circ} \mathrm{C}$. Thereafter, another lumber puncture was done which again revealed motile and flagellate forms of $N$. fowleri which could be cultured. In discussion with the Medicine Department and after reviewing literature, Rifampicin $450 \mathrm{mg}$ was added to the regimen. However, the patient still continued to deteriorate and expired. A request for autopsy was refused by the relatives. 
Figure 1. Growth of amoebae.
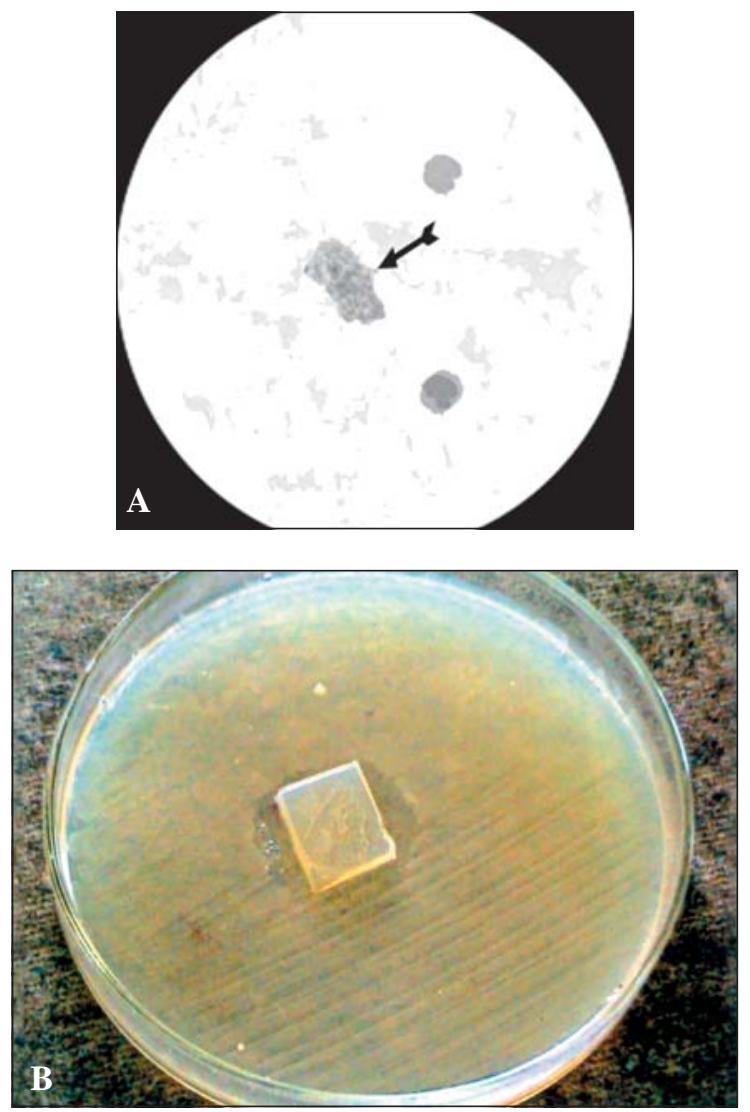

\section{Discussion}

Acute amoebic meningoencephalitis by $N$. fowleri is principally seen in an immunocompetent patient with good health, with prior intimate contact with fresh water, especially in summer months. The portal of entry is via the olfactory mucosa and neuroepithelium. Incubation period is 3-8 days with acute and rapidly fatal course. Patient usually dies in 7-10 days of appearance of symptoms [13]. However in present case no history of swimming could be elicited. Similar cases of PAM without history of swimming have been described by other researchers [14]. However Naegleria infection has also been reported from other sources such as tap water and air $[1,16]$. Our patient had fever, headache, vomiting and drowsiness, followed by coma and death 16 days after admission. The patient was a proved case of ALL in remission and no maintenance therapy.

Initially on admission, differential diagnosis of pyogenic, leukemic, tubercular or cryptococcal meningitis was made on the basis of clinical examination. However on wet mount examination, $N$. fowleri trophozoites and flagellate forms were revealed. Pear shaped flagellate forms of $N$. fowleri swimming in CSF have been reported by other researchers also [17]. Cultural isolation and identification helped us in making accurate diagnosis. Serological testing provides no help in the diagnosis of PAM [17].

The clinical course in our case was slightly prolonged, probably due to the use of drug amphotericin B which may have modified the disease process to some extent. However even after 7 days of therapy CSF still showed $N$. fowleri trophozoites and flagellate forms resulting in ultimately fatal outcome even after addition of rifampicin.

The survival of patients with PAM is rare, even though it has long been known that Naegleria is very sensitive to amphotericin $B$ in vitro and in vivo (mice) [18]. However, amphotericin B was not effective clinically in this case. Most clinicians aim in treatment of PAM is to destroy the amoebae rapidly with full therapeutic dose of amphotericin B because of the fearsome prognosis. However this approach has failed in most of the treatment regimens because fatal outcome might be related to the release of cytopathogenic substances from the disrupted amoebae leading to deposition of complement-activating factors within tissues.

Therefore clinical diagnosis of $N$. fowleri and subsequent patient survival depends most importantly on an initial awareness of the organism and accurate laboratory interpretation of CSF wet mount. Hence diagnosis of PAM should be suspected in every case of purulent meningoencephalitis in which no bacteria are found.

\section{References}

1. Thong Y.H. Primary amoebic meningoencephalitis: 15 years later. Med J Aust 1980;1:352-4.

2. Fowler M., Carter R.F. Acute pyogenic meningitis probably due to Acanthamoeba spp: A preliminary report. Br Med J 1965;2:740-2.

3. Thong Y.H., Rowan-Kelly B., Ferrante A., Shepherd C. Synergism between tetracycline and amphotericin B in experimental amoebic meningoencephalitis. Med J Aust 1978;1:663-4.

4. Thong Y.H., Rowan-Kelly B., Shepherd C., Ferrante A. Growth inhibition of Naegleria fowleri by tetracycline, rifamycin and miconazole. Lancet 1977;2:816.

5. Anderson K., Jamieson A. Primary amoebic encephalitis. Lancet 1972;1:90-2.

6. Apley J., Clarke S.K.R., Roome A.P.C.H. Primary amoebic meningoencephalitis in Britain. BMJ 1970;1:596-9.

7. Brown R.L. Successful treatment of PAM. Arch Intern Med 1991;151:1201-2.

8. Loschiava F., Ventura-Spanolo T., Sessa E. Acute PAM from Naegleria fowleri. Report of a clinical case within a favorable outcome. Acta Neurol (Napoli) 1993;15:333-40.

9. Poungverin N., Jarya P. The fifth nonlethal case of primary amoebic meningoencephalitis. J Med Assoc Thai 1991;74:112-5.

10. Seidel J.S., Harmatz P., Viusvesvera G.S. Successful treatment of PAM. N Eng J Med 1982;306:346-8.

11. Wang A., Kay R., Poon W.S. Successful treatment of amoebic meningoencephalitis in a Chinese living in Hong Kong. Clin Neurol Neurosurg 1993;95:249-52.

12. Singh SN, Patwari AK, Dutta R. Naegleria meningitis. Indian Pediatr 1993;35:101-25.

13. Jain R., Prabhakar S., Modi M., et al. Naegleria meningitis: A rare survival. Neurol India 2002;50:470-2.

14. Shenoy S., Wilson G., Prashanth H.V. et al. Primary meningoencephalitis by Naegleria fowleri; First reported case from Mangalore, South India. J Clin Microbiol 2001;40(1):309.

15. Lawande R.V., Abraham S.N., John I., Egler L.J. Recovery of soil Amebas from the nasal passages of children during the dusty harmattan period in Zaria. Am J Clin Pathol 1979;71(2):201-3.

16. Warhurst D.C., Mann P.G. PAM in bath Spa, England. In: Proceeding of the $2^{\text {nd }}$ International Conference on Biology and pathogenicity of small free-living amoebae. Florida Gainesville, University of Florida. 1980;55.

17. Marshall M.M., Naumovits D., Ortega Y., Sterling C.R. Waterborne protozoan pathogens. Clin Microbiol Rev 1997;10(1):67-85.

18. Carter R.F. Sensitivity to amphotericin B of a Naegleria sp. related from a case of primary amoebic encephalitis. J Clin Path 1969;22:470-4. 